\title{
Editorial
}

\section{Changing scenario of neuropractice}

Biomedical science crucially drives innovation and improvement in medical practice (broad and subspecialties). Furthermore, disciplines, such as clinical epidemiology, have grown and evidencebased medicine ${ }^{[1]}$ as a guideline has come to use. The information used to practice evidence-based medicine contains clinically relevant research often from basic medical sciences, including studies of diagnostic tests, prognostic markers, and the efficacy and safety of therapeutic, rehabilitative, and preventive regimens. In a nutshell, evidence-based medicine consists of integrating individual clinical expertise with the best available external clinical evidence from systematic research.

At one point of time, the aim of neurologists was to arrive at the final diagnosis by artful analysis of the clinical data aided by the least number of laboratory procedures. This was with the belief that the clinician should always keep in mind the primacy of the clinical method and judge the relevance and significance of each laboratory data only in the context of clinical findings. ${ }^{[2]}$ The basic tools had been lumbar puncture for the sample of cerebrospinal fluid, radiology of the skull and spinal column, contrast myelography, pneumoencephalogram (PEG), audiogram, Electronystagmography, and Electromyography. The last few decades have enormously extended the multitude of neuroimaging, advanced biomedical/molecular/genetic methods. The computerized tomography has made an enormous change in the data from scanning available. Magnetic resonance imaging (MRI) has accomplished in obtaining best features of the tissue structure. Furthermore, spiral CT and MRI angiography, along with positron emission tomography (PET scan) have made the final diagnosis easier. Application of light, phase, and electron microscopy in the study of biopsy specimens of muscles, nerves, skin, temporal artery, and brain has made a clear impact in establishing a definitive diagnosis. In short, the armamentarium of neurology has

\begin{tabular}{|l|l|}
\hline \multicolumn{2}{|c|}{ Access this article online } \\
\hline Quick Response Code: & Website: \\
\hline ⿴囗十 & www.ruralneuropractice.com \\
\cline { 2 - 3 } & \\
\hline & \\
\hline
\end{tabular}

substantially expanded for better, faster, and accurate diagnosis and therapeutic management, including stem cell application.

No wonder the new methods are so impressive that they could be substituted (erroneously) for meticulously obtained history and complete clinical neurologic examination. The rigour of making a complete neurologic examination and clinical assessment needs to be meticulously followed and refined, ${ }^{[3]}$ irrespective of the availability of sophisticated technology available at one's fingertips. It is the systematic, sequential, and chronologic pattern adopted that trains the mind for sound approach and not some instant diagnostic or therapeutic solutions or actions at random.

Epilepsy: In nutrition-related problems (deficiency diseases, eg, B12 deficiency), it is now easy to assess the serum levels and in some situations, that is, treatment of epilepsy, where it is necessary to monitor the drug levels (therapeutic drug monitoring) for initiating changes in therapeutic dose adjustments. The choice of antiepileptic drugs has grown and from earlier barbiturates and hydantoin further added with carbamazepin, valproate, oxcarbazepine, gabapentin, topiramate, levetiracetam, lamotrigine, and others with favorable adverse effect profile and minimal pharmacokinetic interaction. ${ }^{[4]}$

CV stroke: Cerebrovascular stroke, one of the top factors and culprits of enormous morbidity and dependence, is commonly attributed to conventional pathophysiologic mechanisms, such as atherothrombosis, cardiogenic causes, and degenerative small-vessel occlusive disease.

Nonatherosclerotic etiologies, such as thrombophilic states and hemoglobinopathies, can now be better identified, but in Indian epidemiologic context, postpartum stroke continues to be a major challenge affecting young women. More light has been thrown on "stroke in young" by advances in the understanding of molecular genetics and cellular metabolic pathways. Cryptogenic stroke (stroke of undetermined etiology) continues to pose a challenge with atrial fibrillation as a factor in causation. ${ }^{[5]}$

With better quality vascular imaging (Early CT, MRI, MR Perfusion Imaging and XeCT/SPECT (at 
certain centers), the diagnostic accuracy is obviously enhanced to facilitate precise interventional approach of thrombolysis - intravenous recombinant t-PA. ${ }^{[6,7]}$

Public awareness in recognition of stroke, availability of ambulance and immediate evacuation of patient to a hospital/stroke center, and training health professionals are of great importance. Prehospital and inhospital delays and lack of stroke center coverage are major issues that may negatively impact stroke care, ${ }^{[8]}$ more so in the southeast Asian context. It is estimated that very few might be receiving thrombolytic therapy because of constraints and the above-mentioned delays, from among the 5\% who qualify for the Thrombolytic Therapy. The time of hospital admission may make the difference in the treatment received whether at weekend or on weekday. ${ }^{[9]}$

Osmotherapy (manitol, glycerol, frusemide) are used in early stages; however, there is no standardization or uniformity for their use. ${ }^{[10]}$

It was found that all subtypes of stroke displayed a significant circadian variation in the time of onset, with rise between 6 am and 12 noon, ${ }^{[11,12]}$ underlying the importance of control of early morning blood pressure surges.

Infections: Pyogenic meningitis, tuberculosis meningitis, viral encephalitis, particularly in children and younger adults continue to pose a clinical challenge; timely hospitalization and intensive care management ${ }^{[13]}$ might contribute toward prompt treatment, better measures, and reduce mortality as compared with those in the earlier decade(s). Syphilis appears to have been replaced by HIV with all its protean manifestations, including central nervous system (CNS) involvement (both disease and drug related). HIV-related neurodegeneration affects different domains of the CNS; recent data provide evidence that cognition and the antiretroviral treatment regimens are reciprocally related in HIV/AIDS. ${ }^{[14]}$

Neurodegenerative Disorders: Accumulation and aggregation of disease-causing proteins is a hallmark of several neurodegenerative disorders, such as Parkinson, Alzheimer, and Huntington diseases. One of the main goals or research in neurodegenerative disorders has been to improve clearance of these accumulated proteins. ${ }^{[15]}$ The relations between the diagnosis and the biological processes are now better understood-epilepsy/ionic balance, multiple sclerosis/inflammation, Alzheimer's disease/amyloid precursor protein processing, spastic paraplegia/endosomal processing, and so on. ${ }^{[16]}$
Dementia that was somewhat an enigma is now better understood as Alzheimer's disease, vascular dementia, Lewy body dementia, and frontotemporal dementia, among which some patients may have a mixed type of Alzheimer and vascular components. With cholinesterase inhibitors (donepezil) and NMDA receptor antagonists, there is a marginal improvement in patients through maximizing functional performance by improving cognition, mood, and behavior as the main goal. ${ }^{[17]}$

There have been additional choices (drugs/surgical) in the management of Parkinson disease, considered a disorder characterized by motor symptoms. Attention is now focused on nonmotor symptoms, such as autonomic dysfunction (gastrointestinal disorders, orthostatic hypotension, sexual dysfunction, urinary incontinence), sleep disorders (restless legs syndrome, periodic limb movements of sleep, excessive daytime somnolence, insomnia, REM sleep behavior disorder), fatigue and anxiety, with botulinum toxin, sildenafil citrate $(50 \mathrm{mg})$, polyethylene glycol as some of the probable useful agents. ${ }^{[18]}$

Neuro Criticare: Neurologic illness and critical care. Critical illness neuropathy ${ }^{[19]}$ exemplifies an acute neuromuscular disorder of severely ill patients, characterized essentially by distal axonal degeneration of motor fibers, causing difficulty in weaning patients from the ventilators.

The usage of neuromuscular blocking agents, such as cisatracurium, in early acute respiratory distress syndrome through possible mechanisms of decreasing the oxygen consumption of respiratory and other muscles, reducing cardiac output, increasing the mixed venous partial pressure of oxygen and increasing the partial pressure of arterial oxygen, and indirectly minimizing manifestations of ventilator-induced lung injury, appear to be the positive developments in a situation of multiorgan failure associated with high mortality.

Neurosurgery: The earlier empirical assessments/ management (often by general surgeon), have become specific, specialized, high-end, and with precision with substantially better results contributed in part by the quality neuroanesthesia techniques.

The above does not purport to be an exhaustive and all inclusive account of the happenings and changes in neuropractice. Suffice to say that the developments in understanding of the etiology, the underlying mechanisms - processes advances in diagnostic and imaging, and improvement in the better choices in 
therapeutics and surgical management have been progressive and in some cases, spectacular.

In the matters of Translational Medicine dimensions, much has been accomplished with some issues ${ }^{[20]}$ as well; clinicians may see perplexing contradictions between the results of observational studies and those of interventional studies.

Globally, the urban-rural divide has apparently reduced because of the phenomenon of urban migration. Although much has been achieved, variability and disparities in health care related to reach and access continue to be a challenge.

ME Yeolekar North Eastern Indira Gandhi Regional Institute of Health and Medical Sciences, Shillong - 793018 , Meghalaya. (Min. of $\mathrm{H}$ and FW), India

Address for correspondence:

Prof. M.E. Yeolekar, North Eastern Indira Gandhi Regional Institute of Health and Medical Sciences, Shillong - 793 018, Meghalaya. (Min. of H and FW), India. E-mail: ayeolekar@yahoo.com

\section{References}

1. Feinstein AR. Evidence-Based Medicine in Oxford Textbook of Medicine. In: Warrel DA, Cox TM, Firth JD, Benz JE, editors. Oxford: 2003. p. 21-4.

2. Victor M, Ropper AH, editors. Principles of Neurology, 7th Ed, New York: McGraw-Hill; 2001. p. 12-41.

3. Yeolekar ME. Redefining and refining the Process of clinical diagnosis. J Assoc Phys Ind 2000;48:769-70.

4. Arif H, Buchsbam R, Pierro J, Whalen M, Sims J, Resor SR Jr, et al. Comparative effectiveness of 10 antiepileptic drugs in older adults with epilepsy. Arch Neurol 2010;67:408-15.

5. Elijovich L, Josephson SA, Fung GL, Smith WS. Intermittent atrial fibrillation may account of large proportion of otherwise cryptogenic stroke: A study of 30-day cardiac event monitors. J Stroke Cerebrovasc Dis 2009; 18:185-9.

6. Padma MV, Singh MB, Bhatia R, Srivastava A, Tripathi M, Shukla G, et al. Hyperacute thrombolysis with IV rtPA of acute ischemic stroke: Efficiency and safety profile of 54 patients as a tertiary referral center in a developing country. Neurol India 2007;55:46-9.

7. Uenaka T, Yoneda Y, Yamamoto S, Hara Y, Yamashita H. Brain imaging modality before systematic thrombolysis for ischemic stroke within three hours. Eur Neurol 2010;64:241-5.

8. Mikael M, Laurent D, Pierre A. Prehospital stroke care: Potential, pitfalls and future. Curr Opin Neurol 2010;23:31-5.

9. Kazley AS, Hillman DG, Johnston KC, Simpson KN. Hospital care for patients experiencing weekend vs weekdays stroke. Arch Neurol 2010;67:39-44.

10. Kalita J, Misra UK, Ranjan R. Prescribing pattern of antiedema therapy in stroke by neurologists and general physicians. Neurol India 2004;52:191-3.

11. Pardiwalla FK, Yeolekar ME, Bakshi SK. Circadian rhythm in acute stroke. J Assoc Physician India 1993; 41:203-4.

12. Elliot WJ. Circadian variation in the timing of stroke onset: A meta analysis. Stroke 1998;29:992-6.

13. Yeolekar ME, Mehta S. ICU care in India: Status and challenges. J Assoc Physicians India 2008;56:221-2.

14. Etternhofer ML, Foley J, Castellon SA, Hinkins, CH, Reciprocal prediction of medication adherence and neurocognition in HIV/AIDS. Neurology 2010;74:1217-22.

15. Dimitri K. Clearance of mutant proteins as a therapeutic target in neurodegenerative diseases. Arch Neurol 2010;67:388-92.

16. Hardy J. Neurological diagnoses identify molecular processes. Arch Neurol 2010;67:400-1.

17. Chertkow H. Diagnosis and treatment of dementia: Introduction. Introducing a series based on the Third Canadian Consensus Conference on the diagnosis and treatment of dementia. CMAJ 2008;178:316.

18. Zesiewicz TA, Sullivan KL, Arnulf I, Chaudhuri KR, Morgan JC, Gronseth GS, et al. Practice parameter: Treatment of nonmotor symptoms of Parkinson disease. Neurology 2010;74:924-31.

19. Schweickert WD, Hall J. ICU-acquired weakness. Chest 2007;131:1541.

20. Ganguli M, Kukull WA. Lost in translation. Arch Neurol 2010;67:107-11.

Source of Support: Nil, Conflict of Interest: None declared.

Announcement

\section{“QUICK RESPONSE CODE” LINK FOR FULL TEXT ARTICLES}

The journal issue has a unique new feature for reaching to the journal's website without typing a single letter. Each article on its first page has a "Quick Response Code". Using any mobile or other hand-held device with camera and GPRS/other internet source, one can reach to the full text of that particular article on the journal's website. Start a QR-code reading software (see list of free applications from http://tinyurl.com/yzlh2tc) and point the camera to the QR-code printed in the journal. It will automatically take you to the HTML full text of that article. One can also use a desktop or laptop with web camera for similar functionality. See http://tinyurl.com/2bw7fn3 or http://tinyurl.com/3ysr3me for the free applications. 